\title{
Findings About Events or Interventions Completion Status
}

National Cancer Institute

\section{Source}

National Cancer Institute. Findings About Events or Interventions Completion Status. NCI

Thesaurus. Code C113367.

A term used to describe the state or condition of the completeness of the findings about events or interventions data. 